\title{
lenstronomy II: A gravitational lensing software ecosystem
}

\author{
Simon Birrer ${ }^{1,2}$, Anowar J. Shajib ${ }^{3,4}$, Daniel Gilman ${ }^{5}$, Aymeric Galan ${ }^{6}$, \\ Jelle Aalbers' ${ }^{1,2}$, Martin Millon ${ }^{6}$, Robert Morgan ${ }^{7,8}$, Giulia Pagano ${ }^{9}$, Ji \\ Won Park $^{1,2}$, Luca Teodori ${ }^{10}$, Nicolas Tessore ${ }^{11}$, Madison Ueland ${ }^{1}$, \\ Lyne Van de Vyvere ${ }^{12}$, Sebastian Wagner-Carena ${ }^{1,2}$, Ewoud Wempe ${ }^{13}$, \\ Lilan Yang ${ }^{14}$, Xuheng Ding ${ }^{15}$, Thomas Schmidt ${ }^{4}$, Dominique Sluse ${ }^{12}$, \\ Ming Zhang ${ }^{16}$, and Adam Amara ${ }^{17}$
}

1 Kavli Institute for Particle Astrophysics and Cosmology and Department of Physics, Stanford University, Stanford, CA 94305, USA 2 SLAC National Accelerator Laboratory, Menlo Park, CA, 94025, USA 3 Department of Astronomy \& Astrophysics, University of Chicago, Chicago, IL 60637, USA 4 Department of Physics and Astronomy, University of California, Los Angeles, CA 90095, USA 5 Department of Astronomy and Astrophysics, University of Toronto, 50 St. George Street, Toronto, ON, M5S 3H4, Canada 6 Institute of Physics, Laboratory of Astrophysics, Ecole Polytechnique Fédérale de Lausanne (EPFL), Switzerland 7 Physics Department, University of Wisconsin-Madison, 1150 University Avenue Madison, WI 53706, USA 8 Legacy Survey of Space and Time Corporation Data Science Fellowship Program, USA 9 Independent Researcher 10 Weizmann Institute, 234 Herzl Street, Rehovot, 7610001 Israel 11 Department of Physics and Astronomy, University College London, Gower Street, London, WC1E 6BT, UK 12 STAR Institute, Université de Liège, Quartier Agora - Allée du six Août, 19c, B-4000 Liège, Belgium 13 Kapteyn Astronomical Institute, University of Groningen, PO Box 800, 9700 AV Groningen, the Netherlands 14 School of Physics and Technology, Wuhan University, Wuhan 430072, China 15 Kavli IPMU (WPI), UTIAS, The University of Tokyo, Kashiwa, Chiba 277-8583, Japan 16 Xinjiang Astronomical Observatory, Chinese Academy of Sciences, 150 Science 1-Street, Urumqi 831001, China 17

DOI: $10.21105 /$ joss.03283 Institute of Cosmology and Gravitation, University of Portsmouth, Portsmouth PO1 3FX, UK

\section{Software}

- Review ¿

- Repository ¿

- Archive ¿

\section{Summary}

lenstronomy is an Astropy-affiliated (Astropy Collaboration et al., 2018, 2013) Python package for gravitational lensing simulations and analyses. lenstronomy was introduced by Birrer \& Amara (2018) and is based on the linear basis set approach by Birrer et al. (2015). The user and developer base of lenstronomy has substantially grown since then, and the software has become an integral part of a wide range of recent analyses, such as measuring the Hubble constant with time-delay strong lensing or constraining the nature of dark matter from resolved and unresolved small scale lensing distortion statistics. The modular design has allowed the community to incorporate innovative new methods, as well as to develop enhanced software and wrappers with more specific aims on top of the lenstronomy API. Through community engagement and involvement, lenstronomy has become a foundation of an ecosystem of affiliated packages extending the original scope of the software and proving its robustness and applicability at the forefront of the strong gravitational lensing community in an open source and reproducible manner. 

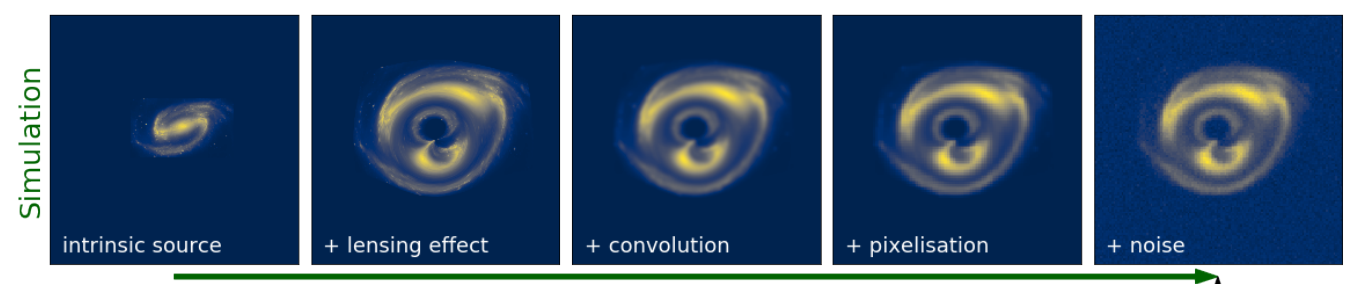

Likelihood(datal
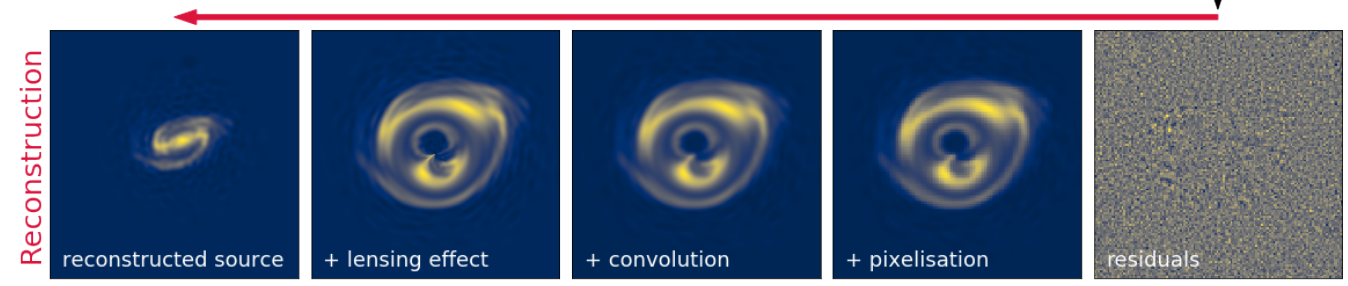

Figure 1: Illustration of the strong gravitational lensing phenomenology and the capabilities of lenstronomy in performing realistic simulations as well as reconstructing lensing and source properties from a given data set. Top row from left to right along the green arrow: A galaxy is lensed around a foreground massive object, becomes highly distorted, and has components appearing multiple times. Observations of this phenomena are limited in resolution (convolution), depending on the detector (pixelation), and are subject to noise. Bottom row from right to left along the red arrow: The inverse problem is solved with a linear basis set in the source morphology maximizing the likelihood of the model given the data.

\section{Background}

Gravitational lensing displaces the observed positions and distorts the shapes of apparent objects on the sky due to intervening inhomogeneous matter along the line of sight. Strong gravitational lensing describes the regime where the background source, such as a galaxy or quasar, is lensed by a massive foreground object, such as another galaxy or cluster of galaxies, to produce multiple images of the source in a highly distorted manner. The top row of Figure 1 illustrates such a process from the intrinsic galaxy to the data product at hand, including the lensing distortions, effects of the instrument, observational conditions, and noise.

Analyses of strong gravitational lensing have provided a wealth of key insights into cosmology and astrophysics. For example, relative time delays of multiply imaged variable sources provided precision measurements on the expansion rate of the Universe (Birrer et al., 2020; Shajib et al., 2020; Wong et al., 2020). Small scale distortions in the lensing signal of resolved sources (Birrer, Amara, et al., 2017; Hezaveh et al., 2016; Vegetti et al., 2012) and unresolved flux ratios (Gilman et al., 2020; Hsueh et al., 2020) constrain the nature of dark matter. Combined strong lensing and kinematic observables constrain the formation and evolution of galaxies (Shajib, Treu, et al., 2021; Sonnenfeld et al., 2015), and the lensing magnification effect provides an otherwise inaccessible angle on the early Universe (Cava et al., 2018; Zheng et al., 2012).

\section{Statement of need}

Strong lensing studies have significantly enhanced, and sometimes challenged, our current fundamental understanding of the Universe. In the near future, with the onset of the nextgeneration ground and space-based wide and deep astronomical imaging (Rubin, Roman, Euclid observatories; Ivezić et al., 2019; Laureijs et al., 2011; Spergel et al., 2013) and interferometric (SKA; Dewdney et al., 2009) surveys, the number of discovered lenses of 
different types will be growing by more than an order of magnitude (Collett, 2015; Oguri \& Marshall, 2010). Such large samples can provide unprecedented statistical precision to stresstest our current understanding and exploit discovery potential. It is key that these demanding studies, at present and in the future, are conducted by reliable software and supported by reproducible and open-source analysis products to provide the most compelling and transparent evidence required to further our physical understanding.

The primary design goal of lenstronomy is to facilitate scientific investigations into the outstanding and most pressing questions in the cosmology and astrophysics community. lens tronomy has been applied throughout its development to the most demanding modeling and inference problems in strong lensing and the software has evolved around the requirements of the scientific applications to facilitate robust analyses. The modular API of the original design of lenstronomy (Birrer \& Amara, 2018) has accommodated the addition of new features. Code review processes in the development phase have led to additional benefits for the user community at large beyond the specific needs of the developer.

lenstronomy provides reliable and well-tested specific functionalities, as well as top-level interfaces, which allow for adaptive and innovative usage in control by the scientific investigator. Guidance for the user community is provided on multiple levels. First, source code is well documented and provided through readthedocs.org. Second, a set of jupyter notebooks are provided in an extension repository. These notebooks demonstrate simplified example use cases, each notebook individually highlighting different specific functionalities of lenstronomy, including a starting guide notebook to introduce the modular design structure of lenstronomy. Third, end-to-end analysis pipelines of some of the published work are publicly available, providing 'real-life' examples at advanced levels.

\section{Track-record of applications}

lenstronomy has been applied in and contributed to more than 30 peer reviewed publications since its first public release in 2018. In particular, lenstronomy has been used to provide state-of-the-art measurements on real data sets, such as: (i) Hubble constant measurements from three quadruly lensed quasars with Hubble Space Telescope (HST) imaging (Birrer et al., 2019, 2016; Shajib et al., 2020), dynamical modeling in the hierarchical analysis by Birrer et al. (2020), and modeling of lensed supernovae (Mörtsell et al., 2020); (ii) inference of small scale dark matter properties from detailed studies of both, resolved imaging (Birrer, Amara, et al., 2017), and unresolved flux ratio statistics (Gilman et al., 2020); (iii) decomposition of quasar and host galaxy light in both, lensed and unlensed cases (Bennert et al., 2021; Ding et al., 2020); (iv) morphological studies of high-redshift sources in the cluster environment (Yang et al., 2021, 2020); (v) internal structure of galaxies (Shajib, Treu, et al., 2021; Shajib, Molina, et al., 2021); (vi) measurements of the weak lensing effect imprinted in Einstein rings (Birrer, Welschen, et al., 2017; Kuhn et al., 2021). Among the studies, some of them have applied a pipeline to uniformly analyse dozens of lenses of different types (Shajib et al., 2019; Shajib, Treu, et al., 2021; Shajib, Molina, et al., 2021), a milestone in moving towards utilizing thousands of lenses in the near future.

Beyond analyzing data, many theoretical studies have been conducted using lenstronomy to investigate statistical robustness in present and anticipated future analyses (Birrer \& Treu, 2019; Ding, Liao, et al., 2021; Li et al., 2021; Millon et al., 2020; Van de Vyvere et al., 2020), as well as to provide forecasts for anticipated future constraints for different science cases (Birrer \& Treu, 2020; Çağan Șengül et al., 2020; Gilman et al., 2019). Particularly, three separate teams participated in the blind time-delay lens modeling challenge (Ding, Treu, et al., 2021) using lenstronomy.

lenstronomy has seen a substantial development and incorporation of innovations and numerical recipes (Birrer, 2021; Galan et al., 2021; Joseph et al., 2019; Shajib, 2019; Tessore 
\& Metcalf, 2015), and has found applications beyond its original aim due to the robust and high-standard design requirements.

\section{Ecosystem of affiliated packages}

lenstronomy has allowed the community to develop third-party analysis products and software products utilizing its core functionalities to provide more targeted and integrated software solutions for a wide range of scientific analyses. These open-source affiliated packages effectively create an ecosystem enhancing the capability of lenstronomy. They provide specified and tested solution for specific scientific investigations, such as plug-ins and direct implementation for innovative source reconstruction algorithms (Galan et al., 2021; SLITronomy; Joseph et al., 2019), gravitational wave lensing computations (lensingGW; Pagano et al., 2020), automated pipelines for gravitational lensing reconstruction (dolphin; Shajib, Treu, et al., 2021), cluster source reconstruction and local perturbative lens modeling (lenstruction; Yang et al., 2020), enhancement in large-scale structure imaging survey simulations (DESC SLSprinkler; LSST Dark Energy Science Collaboration (LSST DESC) et al., 2021), rendering of sub-halos and line-of-sight halos (pyHalo; Gilman et al., 2020), galaxy morphology analysis (galight; Ding et al., 2020), and hierarchical analyses to measure the Hubble constant (hierArc; Birrer et al., 2020). With the rise in popularity and the promises in dealing with ever complex data problems with fast deep-learning methods, dedicated tools for simulating large datasets for applying such methods to strong gravitational lensing (deeplenstronomy; Morgan et al., 2021), (baobab; Park et al., 2021), as well as end-to-end Bayesian Neural Network training and validation packages for Hubble constant measurements (hOrton; Park et al., 2021), and for a hierarchical analysis of galaxy-galaxy lenses (ovejero; Wagner-Carena et al., 2021) have been developed. The affiliated packages make best use of the lenstronomy modules without duplicating source code and make it possible to combine aspects of multiple affiliated packages in one single analysis.

\section{Related open source software}

- lenstronomy (Birrer et al., 2015; Birrer \& Amara, 2018)

- PyAutoLens (Nightingale et al., 2021, 2018)

- gravlens (Keeton, 2011)

- glafic (Oguri, 2010)

- visilens (Spilker et al., 2016)

- PixeLens (Saha \& Williams, 2011)

- GRALE (Liesenborgs et al., 2006)

- lenstool (Jullo \& Kneib, 2009)

\section{Acknowledgements}

Support for this work was provided by the National Science Foundation through NSF AST1716527. AJS was supported by NASA through the STScl grant HST-GO-15320 and by a Dissertation Year Fellowship from the UCLA Graduate Division. This research was supported by the U.S. Department of Energy (DOE) Office of Science Distinguished Scientist Fellow Program. DG is supported by NASA HST-GO-15177. AG, MM LvdV, DS are supported by COSMICLENS: ERC grant agreement No 787886. LT is supported by International HelmholtzWeizmann Research School for Multimessenger Astronomy. MU is supported by KIPAC and the Stanford Summer Research Program. XD is supported by NASA HST-GO-15115. TS is supported by NASA grant HST-GO-15320 and HST-GO-15652. MZ is supported by the 
National Science Foundation of China. AA is supported by a Royal Society Wolfson Fellowship. We are grateful to the user community for valuable feedback and encouragement in continuing the development.

\section{References}

Astropy Collaboration, Price-Whelan, A. M., Sipőcz, B. M., Günther, H. M., Lim, P. L., Crawford, S. M., Conseil, S., Shupe, D. L., Craig, M. W., Dencheva, N., Ginsburg, A., Vand erPlas, J. T., Bradley, L. D., Pérez-Suárez, D., de Val-Borro, M., Aldcroft, T. L., Cruz, K. L., Robitaille, T. P., Tollerud, E. J., ... Astropy Contributors. (2018). The Astropy Project: Building an Open-science Project and Status of the v2.0 Core Package. The Astronomical Journal, 156(3), 123. https://doi.org/10.3847/1538-3881/aabc4f

Astropy Collaboration, Robitaille, T. P., Tollerud, E. J., Greenfield, P., Droettboom, M., Bray, E., Aldcroft, T., Davis, M., Ginsburg, A., Price-Whelan, A. M., Kerzendorf, W. E., Conley, A., Crighton, N., Barbary, K., Muna, D., Ferguson, H., Grollier, F., Parikh, M. M., Nair, P. H., ... Streicher, O. (2013). Astropy: A community Python package for astronomy. Astronomy \& Astrophysics, 558, A33. https://doi.org/10.1051/0004-6361/201322068

Bennert, V. N., Treu, T., Ding, X., Stomberg, I., Birrer, S., Snyder, T., Malkan, M. A., Stephens, A. W., \& Auger, M. W. (2021). A local baseline of the black hole mass scaling relations for active galaxies. IV. Correlations between $M_{\mathrm{BH}}$ and host galaxy $\sigma$, stellar mass, and luminosity. arXiv e-Prints, arXiv:2101.10355. http://arxiv.org/abs/2101.10355

Birrer, S. (2021). Gravitational lensing formalism in a curved arc basis: A continuous description of observables and degeneracies from the weak to the strong lensing regime. arXiv e-Prints, arXiv:2104.09522. http://arxiv.org/abs/2104.09522

Birrer, S., \& Amara, A. (2018). lenstronomy: Multi-purpose gravitational lens modelling software package. Physics of the Dark Universe, 22, 189-201. https://doi.org/10.1016/ j.dark.2018.11.002

Birrer, S., Amara, A., \& Refregier, A. (2017). Lensing substructure quantification in RXJ11311231: a $2 \mathrm{keV}$ lower bound on dark matter thermal relic mass. Journal of Cosmology and Astroparticle Physics, 2017(5), 037. https://doi.org/10.1088/1475-7516/2017/05/037

Birrer, S., Amara, A., \& Refregier, A. (2016). The mass-sheet degeneracy and time-delay cosmography: analysis of the strong lens RXJ1131-1231. Journal of Cosmology and Astroparticle Physics, 2016(8), 020. https://doi.org/10.1088/1475-7516/2016/08/020

Birrer, S., Amara, A., \& Refregier, A. (2015). Gravitational Lens Modeling with Basis Sets. The Astrophysical Journal, 813(2), 102. https://doi.org/10.1088/0004-637X/813/2/102

Birrer, S., Shajib, A. J., Galan, A., Millon, M., Treu, T., Agnello, A., Auger, M., Chen, G. C.-F., Christensen, L., Collett, T., Courbin, F., Fassnacht, C. D., Koopmans, L. V. E., Marshall, P. J., Park, J.-W., Rusu, C. E., Sluse, D., Spiniello, C., Suyu, S. H., ... Van de Vyvere, L. (2020). TDCOSMO. IV. Hierarchical time-delay cosmography joint inference of the Hubble constant and galaxy density profiles. Astronomy \& Astrophysics, 643, A165. https://doi.org/10.1051/0004-6361/202038861

Birrer, S., \& Treu, T. (2020). TDCOSMO V: strategies for precise and accurate measurements of the Hubble constant with strong lensing. arXiv e-Prints, arXiv:2008.06157. http: //arxiv.org/abs/2008.06157

Birrer, S., \& Treu, T. (2019). Astrometric requirements for strong lensing time-delay cosmography. Monthly Notices of the Royal Astronomical Society, 489(2), 2097-2103. https://doi.org/10.1093/mnras/stz2254 
Birrer, S., Treu, T., Rusu, C. E., Bonvin, V., Fassnacht, C. D., Chan, J. H. H., Agnello, A., Shajib, A. J., Chen, G. C.-F., Auger, M., Courbin, F., Hilbert, S., Sluse, D., Suyu, S. H., Wong, K. C., Marshall, P., Lemaux, B. C., \& Meylan, G. (2019). H0LiCOW - IX. Cosmographic analysis of the doubly imaged quasar SDSS $1206+4332$ and a new measurement of the Hubble constant. Monthly Notices of the Royal Astronomical Society, 484(4), 4726-4753. https://doi.org/10.1093/mnras/stz200

Birrer, S., Welschen, C., Amara, A., \& Refregier, A. (2017). Line-of-sight effects in strong lensing: putting theory into practice. Journal of Cosmology and Astroparticle Physics, 2017(4), 049. https://doi.org/10.1088/1475-7516/2017/04/049

Cava, A., Schaerer, D., Richard, J., Pérez-González, P. G., Dessauges-Zavadsky, M., Mayer, L., \& Tamburello, V. (2018). The nature of giant clumps in distant galaxies probed by the anatomy of the cosmic snake. Nature Astronomy, 2, 76-82. https://doi.org/10.1038/ s41550-017-0295-x

Collett, T. E. (2015). The Population of Galaxy-Galaxy Strong Lenses in Forthcoming Optical Imaging Surveys. The Astrophysical Journal, 811(1), 20. https://doi.org/10.1088/ 0004-637X/811/1/20

Çağan Șengül, A., Tsang, A., Diaz Rivero, A., Dvorkin, C., Zhu, H.-M., \& Seljak, U. (2020). Quantifying the line-of-sight halo contribution to the dark matter convergence power spectrum from strong gravitational lenses. Physical Review D, 102(6), 063502. https://doi.org/10.1103/PhysRevD.102.063502

Dewdney, P. E., Hall, P. J., Schilizzi, R. T., \& Lazio, T. J. L. W. (2009). The Square Kilometre Array. IEEE Proceedings, 97(8), 1482-1496. https://doi.org/10.1109/JPROC. 2009.2021005

Ding, X., Liao, K., Birrer, S., Shajib, A. J., Treu, T., \& Yang, L. (2021). Improved timedelay lens modelling and $\mathrm{H}_{0}$ inference with transient sources. 504(4), 5621-5628. https: //doi.org/10.1093/mnras/stab1240

Ding, X., Silverman, J., Treu, T., Schulze, A., Schramm, M., Birrer, S., Park, D., Jahnke, K., Bennert, V. N., Kartaltepe, J. S., Koekemoer, A. M., Malkan, M. A., \& Sanders, D. (2020). The Mass Relations between Supermassive Black Holes and Their Host Galaxies at $1<\mathrm{z}<2$ HST-WFC3. The Astrophysical Journal, 888(1), 37. https://doi.org/10. 3847/1538-4357/ab5b90

Ding, X., Treu, T., Birrer, S., Chen, G. C.-F., Coles, J., Denzel, P., Frigo, M., Galan, A., Marshall, P. J., Millon, M., More, A., Shajib, A. J., Sluse, D., Tak, H., Xu, D., Auger, M. W., Bonvin, V., Chand, H., Courbin, F., ... Williams, L. L. R. (2021). Time delay lens modelling challenge. Monthly Notices of the Royal Astronomical Society, 503(1), 1096-1123. https://doi.org/10.1093/mnras/stab484

Galan, A., Peel, A., Joseph, R., Courbin, F., \& Starck, J.-L. (2021). SLITRONOMY: Towards a fully wavelet-based strong lensing inversion technique. Astronomy \& Astrophysics, 647, A176. https://doi.org/10.1051/0004-6361/202039363

Gilman, D., Birrer, S., Nierenberg, A., Treu, T., Du, X., \& Benson, A. (2020). Warm dark matter chills out: constraints on the halo mass function and the free-streaming length of dark matter with eight quadruple-image strong gravitational lenses. Monthly Notices of the Royal Astronomical Society, 491(4), 6077-6101. https://doi.org/10.1093/mnras/stz3480

Gilman, D., Birrer, S., Treu, T., Nierenberg, A., \& Benson, A. (2019). Probing dark matter structure down to $10^{7}$ solar masses: flux ratio statistics in gravitational lenses with lineof-sight haloes. Monthly Notices of the Royal Astronomical Society, 487(4), 5721-5738. https://doi.org/10.1093/mnras/stz1593

Hezaveh, Y. D., Dalal, N., Marrone, D. P., Mao, Y.-Y., Morningstar, W., Wen, D., Blandford, R. D., Carlstrom, J. E., Fassnacht, C. D., Holder, G. P., Kemball, A., Marshall, P. J., 
Murray, N., Perreault Levasseur, L., Vieira, J. D., \& Wechsler, R. H. (2016). Detection of Lensing Substructure Using ALMA Observations of the Dusty Galaxy SDP.81. The Astrophysical Journal, 823(1), 37. https://doi.org/10.3847/0004-637X/823/1/37

Hsueh, J.-W., Enzi, W., Vegetti, S., Auger, M. W., Fassnacht, C. D., Despali, G., Koopmans, L. V. E., \& McKean, J. P. (2020). SHARP - VII. New constraints on the dark matter free-streaming properties and substructure abundance from gravitationally lensed quasars. Monthly Notices of the Royal Astronomical Society, 492(2), 3047-3059. https://doi.org/ $10.1093 / \mathrm{mnras} / \mathrm{stz} 3177$

Ivezić, Ž., Kahn, S. M., Tyson, J. A., Abel, B., Acosta, E., Allsman, R., Alonso, D., AlSayyad, Y., Anderson, S. F., Andrew, J., Angel, J. R. P., Angeli, G. Z., Ansari, R., Antilogus, P., Araujo, C., Armstrong, R., Arndt, K. T., Astier, P., Aubourg, É., ... Zhan, H. (2019). LSST: From Science Drivers to Reference Design and Anticipated Data Products. The Astrophysical Journal, 873(2), 111. https://doi.org/10.3847/1538-4357/ab042c

Joseph, R., Courbin, F., Starck, J.-L., \& Birrer, S. (2019). Sparse Lens Inversion Technique (SLIT): lens and source separability from linear inversion of the source reconstruction problem. Astronomy \& Astrophysics, 623, A14. https://doi.org/10.1051/0004-6361/ 201731042

Jullo, E., \& Kneib, J.-P. (2009). Multiscale cluster lens mass mapping - I. Strong lensing modelling. 395(3), 1319-1332. https://doi.org/10.1111/j.1365-2966.2009.14654.x

Keeton, C. R. (2011). GRAVLENS: Computational Methods for Gravitational Lensing (p. ascl:1102.003).

Kuhn, F. A., Birrer, S., Bruderer, C., Amara, A., \& Refregier, A. (2021). Combining strong and weak lensing estimates in the Cosmos field. Journal of Cosmology and Astroparticle Physics, 2021(4), 010. https://doi.org/10.1088/1475-7516/2021/04/010

Laureijs, R., Amiaux, J., Arduini, S., Auguères, J.-L., Brinchmann, J., Cole, R., Cropper, M., Dabin, C., Duvet, L., Ealet, A., Garilli, B., Gondoin, P., Guzzo, L., Hoar, J., Hoekstra, H., Holmes, R., Kitching, T., Maciaszek, T., Mellier, Y., ... Zucca, E. (2011). Euclid Definition Study Report. arXiv e-Prints, arXiv:1110.3193. http://arxiv.org/abs/1110.3193

Li, N., Becker, C., \& Dye, S. (2021). The impact of line-of-sight structures on measuring $\mathrm{H}_{0}$ with strong lensing time-delays. Monthly Notices of the Royal Astronomical Society. https://doi.org/10.1093/mnras/stab984

Liesenborgs, J., De Rijcke, S., \& Dejonghe, H. (2006). A genetic algorithm for the nonparametric inversion of strong lensing systems. Monthly Notices of the Royal Astronomical Society, 367(3), 1209-1216. https://doi.org/10.1111/j.1365-2966.2006.10040.x

LSST Dark Energy Science Collaboration (LSST DESC), Abolfathi, B., Alonso, D., Armstrong, R., Aubourg, É., Awan, H., Babuji, Y. N., Bauer, F. E., Bean, R., Beckett, G., Biswas, R., Bogart, J. R., Boutigny, D., Chard, K., Chiang, J., Claver, C. F., Cohen-Tanugi, J., Combet, C., Connolly, A. J., ... Zuntz, J. (2021). The LSST DESC DC2 Simulated Sky Survey. The Astrophysical Journal Supplement Series, 253(1), 31. https://doi.org/10. 3847/1538-4365/abd62c

Millon, M., Galan, A., Courbin, F., Treu, T., Suyu, S. H., Ding, X., Birrer, S., Chen, G. C.-F., Shajib, A. J., Sluse, D., Wong, K. C., Agnello, A., Auger, M. W., Buckley-Geer, E. J., Chan, J. H. H., Collett, T., Fassnacht, C. D., Hilbert, S., Koopmans, L. V. E., ... Van de Vyvere, L. (2020). TDCOSMO. I. An exploration of systematic uncertainties in the inference of $\mathrm{H}_{0}$ from time-delay cosmography. Astronomy \& Astrophysics, 639, A101. https://doi.org/10.1051/0004-6361/201937351

Morgan, R., Nord, B., Birrer, S., Lin, J., \& Poh, J. (2021). deeplenstronomy: A dataset simulation package for strong gravitational lensing. The Journal of Open Source Software, 6(58), 2854. https://doi.org/10.21105/joss.02854 
Mörtsell, E., Johansson, J., Dhawan, S., Goobar, A., Amanullah, R., \& Goldstein, D. A. (2020). Lens modelling of the strongly lensed Type la supernova iPTF16geu. Monthly Notices of the Royal Astronomical Society, 496(3), 3270-3280. https://doi.org/10.1093/ mnras/staa1600

Nightingale, J. W., Dye, S., \& Massey, R. J. (2018). AutoLens: automated modeling of a strong lens's light, mass, and source. MNRAS, 478(4), 4738-4784. https://doi.org/10. $1093 /$ mnras/sty1264

Nightingale, J. W., Hayes, R., Kelly, A., Amvrosiadis, A., Etherington, A., He, Q., Li, N., Cao, X., Frawley, J., Cole, S., Enia, A., Frenk, C., Harvey, D., Li, R., Massey, R., Negrello, M., \& Robertson, A. (2021). PyAutoLens: Open-Source Strong Gravitational Lensing. The Journal of Open Source Software, 6(58), 2825. https://doi.org/10.21105/joss.02825

Oguri, M. (2010). glafic: Software Package for Analyzing Gravitational Lensing (p. ascl:1010.012).

Oguri, M., \& Marshall, P. J. (2010). Gravitationally lensed quasars and supernovae in future wide-field optical imaging surveys. Monthly Notices of the Royal Astronomical Society, 405(4), 2579-2593. https://doi.org/10.1111/j.1365-2966.2010.16639.x

Pagano, G., Hannuksela, O. A., \& Li, T. G. F. (2020). LENSINGGW: a PYTHON package for lensing of gravitational waves. Astronomy \& Astrophysics, 643, A167. https://doi. org/10.1051/0004-6361/202038730

Park, J. W., Wagner-Carena, S., Birrer, S., Marshall, P. J., Lin, J. Y.-Y., Roodman, A., \& LSST Dark Energy Science Collaboration. (2021). Large-scale Gravitational Lens Modeling with Bayesian Neural Networks for Accurate and Precise Inference of the Hubble Constant. The Astrophysical Journal, 910(1), 39. https://doi.org/10.3847/1538-4357/ abdfc4

Saha, P., \& Williams, L. L. R. (2011). PixeLens: A Portable Modeler of Lensed Quasars (p. ascl:1102.007).

Shajib, A. J. (2019). Unified lensing and kinematic analysis for any elliptical mass profile. Monthly Notices of the Royal Astronomical Society, 488(1), 1387-1400. https://doi.org/ $10.1093 / \mathrm{mnras} / \mathrm{stz} 1796$

Shajib, A. J., Birrer, S., Treu, T., Agnello, A., Buckley-Geer, E. J., Chan, J. H. H., Christensen, L., Lemon, C., Lin, H., Millon, M., Poh, J., Rusu, C. E., Sluse, D., Spiniello, C., Chen, G. C.-F., Collett, T., Courbin, F., Fassnacht, C. D., Frieman, J., ... Zhang, Y. (2020). STRIDES: a 3.9 per cent measurement of the Hubble constant from the strong lens system DES J0408-5354. Monthly Notices of the Royal Astronomical Society, 494(4), 6072-6102. https://doi.org/10.1093/mnras/staa828

Shajib, A. J., Birrer, S., Treu, T., Auger, M. W., Agnello, A., Anguita, T., Buckley-Geer, E. J., Chan, J. H. H., Collett, T. E., Courbin, F., Fassnacht, C. D., Frieman, J., Kayo, I., Lemon, C., Lin, H., Marshall, P. J., McMahon, R., More, A., Morgan, N. D., ... Walker, A. R. (2019). Is every strong lens model unhappy in its own way? Uniform modelling of a sample of 13 quadruply+ imaged quasars. Monthly Notices of the Royal Astronomical Society, 483(4), 5649-5671. https://doi.org/10.1093/mnras/sty3397

Shajib, A. J., Molina, E., Agnello, A., Williams, P. R., Birrer, S., Treu, T., Fassnacht, C. D., Morishita, T., Abramson, L., Schechter, P. L., \& Wisotzki, L. (2021). High-resolution imaging follow-up of doubly imaged quasars. Monthly Notices of the Royal Astronomical Society, 503(2), 1557-1567. https://doi.org/10.1093/mnras/stab532

Shajib, A. J., Treu, T., Birrer, S., \& Sonnenfeld, A. (2021). Dark matter haloes of massive elliptical galaxies at $\mathrm{z} \sim 0.2$ are well described by the Navarro-Frenk-White profile. Monthly Notices of the Royal Astronomical Society, 503(2), 2380-2405. https://doi.org/10.1093/ mnras/stab536 
Sonnenfeld, A., Treu, T., Marshall, P. J., Suyu, S. H., Gavazzi, R., Auger, M. W., \& Nipoti, C. (2015). The SL2S Galaxy-scale Lens Sample. V. Dark Matter Halos and Stellar IMF of Massive Early-type Galaxies Out to Redshift 0.8. The Astrophysical Journal, 800(2), 94. https://doi.org/10.1088/0004-637X/800/2/94

Spergel, D., Gehrels, N., Breckinridge, J., Donahue, M., Dressler, A., Gaudi, B. S., Greene, T., Guyon, O., Hirata, C., Kalirai, J., Kasdin, N. J., Moos, W., Perlmutter, S., Postman, M., Rauscher, B., Rhodes, J., Wang, Y., Weinberg, D., Centrella, J., ... Shaklan, S. (2013). Wide-Field InfraRed Survey Telescope-Astrophysics Focused Telescope Assets WFIRSTAFTA Final Report. arXiv e-Prints, arXiv:1305.5422. http://arxiv.org/abs/1305.5422

Spilker, J. S., Marrone, D. P., Aravena, M., Béthermin, M., Bothwell, M. S., Carlstrom, J. E., Chapman, S. C., Crawford, T. M., de Breuck, C., Fassnacht, C. D., Gonzalez, A. H., Greve, T. R., Hezaveh, Y., Litke, K., Ma, J., Malkan, M., Rotermund, K. M., Strandet, M., Vieira, J. D., ... Welikala, N. (2016). ALMA Imaging and Gravitational Lens Models of South Pole TelescopeSelected Dusty, Star-Forming Galaxies at High Redshifts. The Astrophysical Journal, 826, 112. https://doi.org/10.3847/0004-637X/826/2/112

Tessore, N., \& Metcalf, R. B. (2015). The elliptical power law profile lens. Astronomy \& Astrophysics, 580, A79. https://doi.org/10.1051/0004-6361/201526773

Van de Vyvere, L., Sluse, D., Mukherjee, S., Xu, D., \& Birrer, S. (2020). The impact of mass map truncation on strong lensing simulations. Astronomy \& Astrophysics, 644, A108. https://doi.org/10.1051/0004-6361/202038942

Vegetti, S., Lagattuta, D. J., McKean, J. P., Auger, M. W., Fassnacht, C. D., \& Koopmans, L. V. E. (2012). Gravitational detection of a low-mass dark satellite galaxy at cosmological distance. Nature, 481(7381), 341-343. https://doi.org/10.1038/nature10669

Wagner-Carena, S., Park, J. W., Birrer, S., Marshall, P. J., Roodman, A., Wechsler, R. H., \& LSST Dark Energy Science Collaboration. (2021). Hierarchical Inference with Bayesian Neural Networks: An Application to Strong Gravitational Lensing. The Astrophysical Journal, 909(2), 187. https://doi.org/10.3847/1538-4357/abdf59

Wong, K. C., Suyu, S. H., Chen, G. C.-F., Rusu, C. E., Millon, M., Sluse, D., Bonvin, V., Fassnacht, C. D., Taubenberger, S., Auger, M. W., Birrer, S., Chan, J. H. H., Courbin, F., Hilbert, S., Tihhonova, O., Treu, T., Agnello, A., Ding, X., Jee, I., ... Meylan, G. (2020). HOLiCOW XIII. A 2.4 per cent measurement of $\mathrm{H}_{0}$ from lensed quasars: $5.3 \sigma$ tension between early- and late-Universe probes. Monthly Notices of the Royal Astronomical Society, 498(1), 1420-1439. https://doi.org/10.1093/mnras/stz3094

Yang, L., Birrer, S., \& Treu, T. (2020). A versatile tool for cluster lensing source reconstruction - I. Methodology and illustration on sources in the Hubble Frontier Field Cluster MACS J0717.5+3745. Monthly Notices of the Royal Astronomical Society, 496(3), 2648-2662. https://doi.org/10.1093/mnras/staa1649

Yang, L., Roberts-Borsani, G., Treu, T., Birrer, S., Morishita, T., \& Bradač, M. (2021). The evolution of the size-mass relation at $z=1-3$ derived from the complete Hubble Frontier Fields data set. Monthly Notices of the Royal Astronomical Society, 501(1), 1028-1037. https://doi.org/10.1093/mnras/staa3713

Zheng, W., Postman, M., Zitrin, A., Moustakas, J., Shu, X., Jouvel, S., Høst, O., Molino, A., Bradley, L., Coe, D., Moustakas, L. A., Carrasco, M., Ford, H., Benítez, N., Lauer, T. R., Seitz, S., Bouwens, R., Koekemoer, A., Medezinski, E., ... van der Wel, A. (2012). A magnified young galaxy from about 500 million years after the Big Bang. Nature, 489(7416), 406-408. https://doi.org/10.1038/nature11446 